\title{
Doubly Compacted Schwinger Model
}

\author{
Román Linares* \\ Departamento de Física de Altas Energías, Instituto de Ciencias Nucleares, \\ Universidad Nacional Autónoma de México. Apartado Postal 70-543, 04510, México D.F. \\ E-mail: 'Iinares@nuclecu.unam.mx.'
}

ABstract: We summarize the complete exact solution of the doubly compacted Schwinger model (DCSM), defined by the condition that the domain of the electromagnetic degree of freedom $c=\frac{1}{L} \int_{0}^{L} d x A_{1}$ is such that $-\bar{c}<c<+\bar{c}$. The results are contrasted with the standard situation, where $-\infty<c<+\infty$, which we call the non-compact case (NCSM). Both theories are also compacted in a circle of length $L$ for the space variable $x$.

\section{Introduction}

The motivation for having a compact domain in the electromagnetic variable $c$ arises from the loop formulation of gauge theories [i]1, applied to this model. In this approach all the information about the theory is encoded in terms of variables, which are gauge invariant under small and large gauge transformations. In $1+1$ dimensions the basic variable for the electromagnetic degrees of freedom is the holonomy $T=\exp i e c L$, which exhibits the property that the choice of $c$ in the interval $-\bar{c}<c<\bar{c}$, with $\bar{c}=\frac{\pi}{e L}$, is sufficient to describe the physics of the problem [2].

This feature of the compacted model leads to a solution which has different properties from those arising in the non-compact case. Among the numerous papers on the NCSM [3i-1] , we heavily relay on the work of Iso and Murayama [4int]. The details of the solution in the DCSM can be found in Ref. [iñ

\section{Solution of the model}

The Schwinger model is masless QED in $1+1$ dimensions. In a standard notation the Lagrangian is

$$
\mathcal{L}=-\frac{1}{4} F_{\mu \nu} F^{\mu \nu}+\bar{\psi} \gamma^{\mu}\left(i \partial_{\mu}-e A_{\mu}\right) \psi
$$

*Luis F. Urrutia (urrutia@nuclecu.unam.mx) and J. David Vergara (vergara@nuclecu.unam.mx) where we adopt periodic (antiperiodic) bundary conditions for the fields $A_{\mu}(\psi)$. The above Lagrangian is invariant under the gauge transformations $\psi \rightarrow e^{i e \alpha(x, t)} \psi, \quad A_{\mu} \rightarrow A_{\mu}-\partial_{\mu} \alpha(x, t)$. There are two families of gauge transformations: (i) those continuously connected to the identity, called small gauge transformations, characterized by the function $\alpha(x, t)=b(t) e^{i 2 \pi n x / L}$ (ii) the second family corresponds to the so called large gauge transformations, which are generated by the non-periodic functions $\alpha(x)=\frac{2 \pi n}{e L} x, n=$ $\pm 1, \pm 2, \ldots$. The compactification condition upon $c$ means that two values of $c$ differing by $\frac{2 \pi}{e L}$ must be identified. In this way $c$ is invariant under both types of gauge transformations, as opposed to the non-compact case where $c \rightarrow c+\frac{2 \pi}{e L}$ under large gauge transformations.

After the standard canonical analysis of the Lagrangian density $(2.1)$, describing the configuration space variables $A_{0}, A_{1}$ and $\psi$, we obtain

$$
\begin{aligned}
\mathcal{H}= & \frac{1}{2} E^{2}+i \psi^{\dagger} \sigma_{3}\left(\partial_{1}+i e A_{1}\right) \psi \\
& -A_{0}\left(\partial_{1} E-e \psi^{\dagger} \psi\right), \\
\Pi_{0} \approx & 0,
\end{aligned}
$$

where the corresponding canonical momenta are $\Pi_{0}, \Pi_{1}=F_{01}=E$ and $\Pi_{\psi}=-i \psi^{*}$. Conservation in time of the primary constraint $\Pi_{0} \approx 0$ leads to the the Gauss law constraint $\mathcal{G}=\partial_{1} E-$ $e \psi^{\dagger} \psi \approx 0$. There are no additional constraints.

The quantization proceeds in a way completely similar to the standard case. We choose 
the Coulomb gauge $\frac{\partial A_{1}}{\partial x}=0$, which means that $A_{0}=0$ and $c=A_{1}(t)$. The latter equality corresponds to the zero mode of the electromagnetic potential and it is the only electromagnetic degree of freedom that remains. We fix the gauge in the temporal component of the electromagnetic potential, together with $\Pi_{0}=0$. The only remaining constraint $\mathcal{G}$ is first class and it is imposed strongly upon the physical states of the system. The implementation of the zero-mode of the Gauss law, as a constraint determining the physical sates, implies that these must be states of zero electric charge.

The first step in the quatization is the construction of the fermionic Fock space in an external electromagnetic field. Here a main difference arises because, in the DCSM, it is possible to introduce the conserved and gauge invariant chiral charge

$\bar{Q}_{5}=\lim _{s \rightarrow 0} \sum_{n=-\infty}^{+\infty} \frac{1}{\left|\lambda \epsilon_{n}\right|^{s}}\left(a_{n}^{\dagger} a_{n}-b_{n}^{\dagger} b_{n}\right)+\frac{e c L}{\pi}$,

with eigenvalues $2 N, N=0, \pm 1, \ldots$, where a $\zeta$-regularization is suggested. The one-particle energy eigenvalues of $h_{F}$, where $\mathcal{H}_{F}=\psi^{\dagger} h_{F} \psi$, are $\epsilon_{n}=\frac{2 \pi}{L}\left(n+\frac{1}{2}-\frac{e L}{2 \pi} c\right) \equiv \frac{2 \pi n}{L}+\mathcal{C}$, which are fully gauge invariant in virtue of the compactification process. Here, $a_{n}$ and $b_{n}$ denote generically the fermionic creation and annihilation operators for each chirality. Under both type of gauge tranformations, they change as $a_{n} \rightarrow$ $\mathrm{e}^{i e \alpha(0)} a_{n}, \quad b_{n} \rightarrow \mathrm{e}^{i e \alpha(0)} b_{n}$. This establishes another difference with respect to the NCSM. In the latter situation, large gauge transformations lead to $a_{n} \rightarrow a_{n+1}, b_{n} \rightarrow b_{n+1}$. States of minimum energy are Dirac-type vacuums with all negative energy levels filled,

$$
\left|\mathcal{E}_{N}, 2 N\right\rangle=\prod_{n=-\infty}^{N-1} a_{n}^{\dagger}|0\rangle \otimes \prod_{m=N}^{\infty} b_{m}^{\dagger}|0\rangle
$$

each having chiral charge $2 N$ and energy $\mathcal{E}_{N}(c)=$ $\frac{2 \pi}{L}\left\{\left(N-\frac{e c L}{2 \pi}\right)^{2}-\frac{1}{12}\right\}$. These states are related by operators of the form $j_{+-}^{n m} \equiv a_{n}^{\dagger} b_{m}, j_{-+}^{n m} \equiv$ $b_{n}^{\dagger} a_{m}$. These operators can be introduced in both formulations of the model (compact and no compact) however in the NCSM one can understand the relation among the states $\left(\begin{array}{l}12-\overline{4} \\ -2\end{array}\right)$ as large gauge transformations. Since in the DCSM the states are fully gauge invariant we do not have this interpretation here. Our compactification condition implies that the state with $N=0$ is the true vacuum at this stage.

The excited states are constructed by using the following current operators

$$
\begin{aligned}
& \psi_{1}^{\dagger}(x) \psi_{1}(x)=\frac{1}{L} \sum_{n=-\infty}^{+\infty} \mathrm{e}^{-\frac{2 \pi i n}{L} x} j_{+}{ }^{n} \\
& \psi_{2}^{\dagger}(x) \psi_{2}(x)=\frac{1}{L} \sum_{n=-\infty}^{+\infty} \mathrm{e}^{+\frac{2 \pi i n}{L} x} j_{-}{ }^{n}
\end{aligned}
$$

In terms of the corresponding zero modes, we have $Q=j_{+}{ }^{0}+j_{-}{ }^{0}, \bar{Q}_{5}=j_{+}{ }^{0}-j_{-}{ }^{0}+\frac{e c L}{\pi}$. The operators $j_{ \pm}^{n}, n \geq 1$ annihilate the states (12.4). Finally, the fermionic Fock space in the background electromagnetic field will consist of all the local vacuums $(2.4)$, together with all possible states constructed from them by the application of an arbitrary number of the current operators $\left(j_{ \pm}{ }^{n}\right)^{\dagger}, n=1,2, \ldots$ defined above. The regularized current algebra on this fermionic Fock space is given by

$$
\begin{gathered}
{\left[j_{+}{ }^{n},\left(j_{+}{ }^{m}\right)^{\dagger}\right]=} \\
n \delta_{m, n}, \quad\left[j_{-}{ }^{n},\left(j_{-}{ }^{m}\right)^{\dagger}\right]=n \delta_{m, n} \\
{\left[j_{+}{ }^{n}, j_{-}{ }^{m}\right]=0 .}
\end{gathered}
$$

Taking into account the spectrum of the system, together with the way in which the Fock space has been constructed, the fermionic Hamiltonian in the external field can be rewritten as the following Sugawara transformed expression

$$
H_{F}=\mathcal{E}_{N}(c)+\frac{2 \pi}{L} \sum_{n>0}\left(\left(j_{+}^{n}\right)^{\dagger} j_{+}^{n}+\left(j_{-}^{n}\right)^{\dagger} j_{-}^{n}\right) .
$$

Next we consider the quantization of the electromagnetic Fourier modes $A_{m}, E_{m}, m=0, \pm 1, \ldots$. The commmutator $\left[E(x), \psi_{\alpha}(y)\right]=0$ leads to

$$
\begin{aligned}
{\left[E_{m}, a_{n}\right] } & =\frac{i e}{2 \pi m}\left(a_{n}-a_{n+m}\right), \quad m \neq 0, \\
{\left[E_{0}, a_{n}\right] } & =0
\end{aligned}
$$

together with an analogous relation for the $b$ 's. The above commutators arise because in the expansion of the fermionic fields in Fourier modes we have used the wave functions in the electromagnetic field, instead of pure plane waves. The 
remaining commutators are the expected ones. The required commutation relations are satisfied by choosing

$$
\begin{aligned}
E_{m} & =\frac{1}{i L} \frac{\partial}{\partial A_{m}}-\frac{e}{2 \pi i m}\left(j_{+}{ }^{m}+\left(j_{-}{ }^{m}\right)^{\dagger}\right), m \neq 0, \\
E_{0} & =\frac{1}{i L} \frac{\partial}{\partial c} .
\end{aligned}
$$

The solution of Eq. $(\underline{2} \cdot \overline{8}-\bar{s})$ leads to the following dependence of the fermionic operators upon the non-zero electromagnetic modes

$$
a_{m}=\exp \left(-\frac{e L}{2 \pi} \sum_{k \neq 0} \frac{1}{k} A_{k}\right) \bar{a}_{m},
$$

where $\bar{a}_{m}$ are new fermionic operators which are independent of the gauge field $A_{k}$. With the realization (2.9i), the non-zero modes of the Gauss law reduce to $\mathcal{G}_{m}=\frac{2 \pi m}{L} \frac{\partial}{\partial A_{m}} \approx 0, \quad m \neq 0$. Then we conclude that the wave functions of the system must be of zero electric charge and also independent of the modes $A_{m}, m \neq 0$. Our expression for the Gauss law is somewhat different from the one obtained in $\left[\begin{array}{c}\overline{4} \\ 4\end{array}\right]$, though the final results are equivalent. In this way we conclude that any further compactification in the electromagnetic modes $A_{m}$ is irrelevant and also that the compactification of $c$ does not have any further effect in the fermionic degrees of freedom of the model. The next step is to write the full Hamiltonian making use of the Gauss law. This allows us to rewrite the operators $E_{m}, m \neq 0$ in terms of the currents introduced in (2.5). The resulting Hamiltonian is subsequently diagonalized by a Bogoliubov transformation [신, leading to

$$
\begin{aligned}
H & =\frac{\pi}{2 L}\left(\bar{Q}_{5}-\frac{e c L}{\pi}\right)^{2}-\frac{1}{2 L}\left(\frac{\partial}{\partial c}\right)^{2} \\
& +\sum_{n>0} \frac{E_{n}}{n}\left(\left(j_{+}^{n}\right)^{\dagger} j_{+}^{n}+\left(j_{-}^{n}\right)^{\dagger} j_{-}^{n}\right),
\end{aligned}
$$

up to an infinite constant. In this equation $E_{n}=$ $\sqrt{\left(\frac{2 \pi n}{L}\right)^{2}+M^{2}}$, where $M=\frac{e}{\sqrt{\pi}}$. The Bogoliubov rotation is a unitary transformation $U_{B}$ which commutes with the electric and axial charge operators. To construct the Hilbert space of the full theory we will start from the states $|N\rangle_{B}=$ $\left|\mathcal{E}_{N}, 2 N\right\rangle$, arising from Eq.(2). (T) (The subscript $B$ in any ket is to remind us that such vector is written in the Bogoliubov rotated frame). As in the

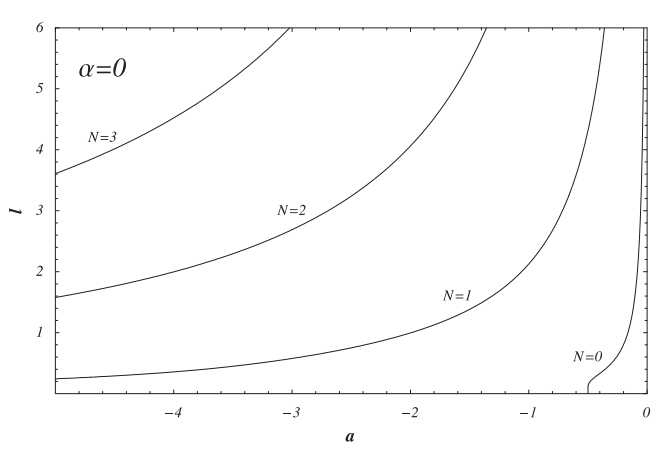

Figure 1: The above curves provide the numerical solution for the parameter $a_{0, N}(l)$, for $N=0 \ldots 3$ and a given value $l$. The energies are $E_{0, N, 0}=$ $-\left(e / \pi^{1 / 2}\right) a_{0, N}$

non-compact case, each mode decouples in such a way that the Schroedinger equation $H \Delta=E \Delta$ is solved by $\Delta=\Pi_{n} \Delta_{n}$, where $H_{n} \Delta_{n}=E_{n} \Delta_{n}$ and $E=\sum E_{n}$. The general strategy to construct the Hilbert space will be to start from the zero modes $F_{N}(c) \times|N\rangle_{B}$ and to subsequently apply all possible combinations of the raising operators $\left(j_{ \pm}{ }^{m}\right)^{\dagger}$ to them.

The zero modes correspond to the choice $\mid$ ground; $N\rangle=F_{N}(c) \times|N\rangle_{B}$, where wave functions $F_{N}(c)$ satisfy the following Schroedinger equation

$$
\begin{aligned}
& \left(-\frac{1}{2 L}\left(\frac{\partial}{\partial c}\right)^{2}+\frac{e^{2} L}{2 \pi}\left(\frac{2 \pi N}{e L}-c\right)^{2}\right) F_{N}(c) \\
& =E_{N, 0} F_{N}(c)
\end{aligned}
$$

subjected to the boundary conditions

$$
\left.F_{N}\right|_{c=-\bar{c}}=\left.F_{N}\right|_{c=+\bar{c}},\left.\quad \frac{\partial F_{N}}{\partial c}\right|_{c=-\bar{c}}=\left.\frac{\partial F_{N}}{\partial c}\right|_{c=+\bar{c}}
$$

arising from the compactification procedure. This choice should be contrasted with that of Manton, written in Eqs. (3.15) of Ref. [i, $\overline{6}]$. The above Schroedinger equation (2. $\overline{1} \overline{1} \overline{2})$ and boundary conditions $(2 . \overline{1} \cdot \overline{3}$ i) lead to energies which are not any more given by the characteristic equally spaced harmonic oscillator spectrum, as it is the case in the NCSM.

The energy eigenvalues are parametrized in the form $E_{\alpha, N, 0}=-\frac{e a_{\alpha, N}}{\sqrt{\pi}}$, where $\alpha$ labels the resulting eigenvalues. A numerical evaluation of $a_{\alpha, N}$ as a function of $l=\frac{e L}{\pi^{3 / 2}}$ is presented in 


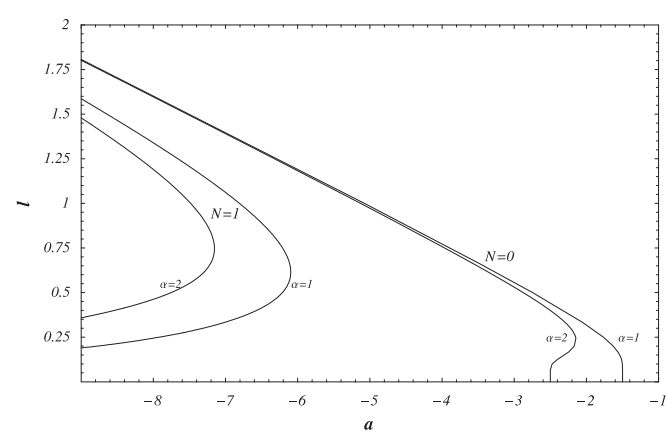

Figure 2: The numerical solution of $a_{\alpha, N}(l)$, with $N=0,1$ and $\alpha=1,2$, for a given value of $l$, is given. The energies are $E_{\alpha, N, 0}=-\left(e / \pi^{1 / 2}\right) a_{\alpha, N}$

Figs.(1) and (2). From the numerical calculation we find that the minimun value of $E_{0, N, 0}$ correspond to $N=0$. Thus, in the DCSM the physical, non-degenerated, vacuum of the theory is $|0,0,0\rangle_{B}=F_{0}(c) \times|0\rangle_{B}$.

The excited states are obtained by appliying the creation operators $\left(j_{ \pm}{ }^{m}\right)^{\dagger}$ to the zero modes of the problem. Each individual action raises the energy by $E_{m}$. The excited states will be labeled by $\left|\alpha, N, N_{1}, \ldots, N_{k}, \ldots\right\rangle_{B}$, where $N_{k}$ is the the total number of times that the operators $\left(j_{ \pm}{ }^{k}\right)^{\dagger}$ have been applied to the corresponding minimun energy state. This is the ocuppation number of the $k$-level. The total energy of the above state is given by

$$
E_{\alpha, N, N_{1}, N_{2} \ldots N_{k}, \ldots}=E_{\alpha, 0, N}+\sum_{k>0} N_{k} E_{k}
$$

The fact that $\tilde{Q}_{5}$ is conserved in the full Hilbert space of the model is a direct consequence of the way in which the Hilbert space has been constructed and can be proved accordingly.

\section{Final comments}

Motivated by the loop-space formulation of $1+1$ massless QED, we have exactly solved the doubly compactified Schwinger model, where both the spatial coordinate $x$ together with the electromagnetic degree of freedom $c$ are compactified. The first consequence of the compactification of $c$ is that the surviving electromagnetic degree of freedom is invariant under both small and large gauge transformations (LGT). This implies the invariance of the individual eigenvalues $\epsilon_{n}$ of the fermionic Fock space, together with the phase transformation $a_{n} \rightarrow \mathrm{e}^{i e \alpha(0)} a_{n}, \quad b_{n} \rightarrow \mathrm{e}^{i e \alpha(0)} b_{n}$, for the fermionic operators $a_{n}, b_{n}$, under LGT. This has to be contrasted with the standard case, where $\epsilon_{n} \rightarrow \epsilon_{n+1}, a_{n} \rightarrow a_{n+1}, b_{n} \rightarrow b_{n+1}$ under LGT. The next consequence has to do with the definition of the total chiral charge $\bar{Q}_{5}$, which is conserved and independent of the electromagnetic degree of freedom $c$ in both cases. This charge $\bar{Q}_{5}$ has the invariance(non-invariance) under LGT in the compact(non-compact) case, in virtue of the transformation properties of the fermionic operators. Thus, the compactification requirement allows us to have the conservation of the electric charge together with the modified chiral charge. That is to say, we have neither vector nor axial-vector charge anomalies. Nevertheless, the axial current anomaly is still present due to the impossibility of constructing a local, fully gauge invariant current $\bar{J}_{\mu 5}$, which reproduces the conserved axial charge $\bar{Q}_{5}$.

Next we discuss the spectra of the models. In the standard non-compact case we have an infinite set of sectors labeled by the integer $N$, which are connected by LGT. The corresponding zero modes in each sector have energies given by $(n+1 / 2) e / \sqrt{\pi}, n=0,1,2, \ldots$, independently of the label $N$, thus been infinitely degenerated. It is precisely this property that requires the introduction of the $\theta$-vacuum. In the compact case, the sectors labeled by $N$, corresponding here to the eigenvalues $2 N$ of $\bar{Q}_{5}$, are also present. They are connected through the operators $j_{+-}, j_{-+}$. Nevertheless, due to the boundary conditions $\left(i \overline{1} 3_{1}\right)$, the corresponding zero modes energies depend upon the label $N$ and are non-degenerated as can be seen in Figs.(1) and (2). In fact, the lowest energy state corresponding to the $N=0$ sector is the ground state of the model. Thus, no $\theta$-vacuum is required in the present case. The exited states are constructed by the same procedure in both cases by applying the raising operators $\left(j_{ \pm}{ }^{m}\right)^{\dagger}$ to the zero mode states. Nevertheless, their action produces both eigenvectors and eigenvalues which are different with respect to the non-compact case. The non-equally spaced spectra of the zero mode does not lead to a particle interpretation of the compact model, as it 
is the case in the non-compact situation.

For a given $L \neq 0$, the boundary conditions

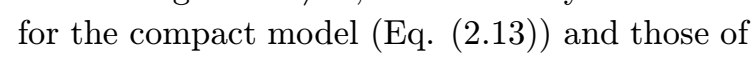
the non-compact case ( Eqs. (3.15) of Ref. [i,

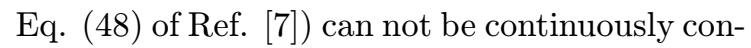
nected between each other. Thus, neither model can be obtained from the other through an adequate limiting process.

\section{Acknowledgments}

Partial support from the grants CONACyT 32431-

E and DGAPA-UNAM-IN100397 is acknowledged.

\section{References}

[1] R. Gambini and J. Pullin, Loops, knots, gauge theories and quantum gravity (Cambridge: Cambridge Univ. Press 1996).

[2] R. Gambini, H. Morales, L. F. Urrutia and J.D. Vergara, Phys. Rev. D $\mathbf{5 7}(1998)$ 3711'.

[3] J. Schwinger, Phys. Rev. 125, 397 (1962); ibid 128,2425 (1962). For a recent review of the standard Schwinger model see for example C. Adam, Anomaly and Topological aspects of twodimensional quantum electrodynamics, Dissertation, Universitat Wien, october 1993. See also: C. Adam, CZech J. Phys. 48, 9 (1998); C. Adam, Z. Phys. C63, 169 (1994); C. Adam, R.A. Bertlmann and P. Hofer, Riv. 'Nuovo Cim.'

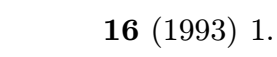

_ 4$]$ S. Iso and H. Murayama, 'Prog. Theor. Phys.



[5] R. Linares, L.F. Urrutia and J.D. Vergara, The Hamiltonian solution of the doubly compactified Schwinger model, in preparation.

[6] N.S. Manton, 'Ann. Phys. $(N Y) \mathbf{1 5 9}(1985) 220$.

[7] R. Link, 'Phys. Rev. D'D42 (1990) 210 . 